\title{
Development and Evaluation of Greening Materials Using Biodegradable Water-soluble Polymers
}

\author{
Y. Katsumura and O. Yoshimura \\ Kanazawa Institute of Technology, 3-1 Yatsukaho, Hakusan, Ishikawa 924-0838 Japan \\ * Corresponding author: E-mail: b6901110@planet.Kanazawa-it.ac.jp
}

\begin{abstract}
An environmentally friendly greening material was fabricated using water-soluble, biodegradable polyaspartic acid derived from biomass. Polyaspartic acid was cross-linked with 1,4diaminobutane, where the best water absorption was achieved when the cross-linking agent/polymer ratio was $20 \%$. Moreover, germination tests of soil employing the water-absorbing polymer showed promoted elongation growth and suppressed axial length dispersion in tested plants.
\end{abstract}

Key words: Water-absorbing polymer, Polyaspartic acid, Greening material, Biomass

\section{INTRODUCTION}

Water-absorbing polymers can absorb amounts of water several hundred times their own weight without dissolving [1]. The most well-known example is sodium polyacrylate, whose main chain consists of methylene ($\mathrm{CH}_{2}-$ ), methine (-CH-), and carboxyl units. Therefore, it does not decompose in the environment and is watersoluble. Sodium acrylate-based polymers are synthesized from non-biodegradable, water-soluble petroleum resources, which may lead to the depletion of petroleum resources and their diffusion/accumulation in the environment. Moreover because a large amount of water and filth are included in the utilization process, a significant loss occurs during their incineration, and their recycling is difficult [2].

Water-absorbing polymers can serve as a greening material because they contain substances necessary for plant growth, such as water and nutrient sources, and can be applied to growth beds. These polymers could also help plants retain water so they grow from well-drained soil or soil in dry environments that receive little water [3]. Despite these benefits, existing water-absorbing polymers are difficult to decompose and are watersoluble; therefore, environmentally friendly greening materials are desirable. The objective of this study was to synthesize greening materials from sources with low environmental impact.

Polyaspartic acid (PAsp) is a polyamino acid with its repeating unit consisting of a carboxylic acid and an amide. PAsp is also a biodegradable polycarboxylic acid that could replace sodium acrylate-based polymers [4]. Furthermore, it can be facilely fabricated from aspartic acid without the need of complex monomer syntheses, beginning with aspartic acid being converted into polysuccinimide (PSI), a PAsp precursor, through simple heating (Fig. 1) [5]. Large amounts of aspartic acid can be synthesized from biomass via fumaric acid at low costs and without petroleum resources.

In this study, PAsp was cross-linked with the diamine 1,4-diaminobutane (DAB) to from a water-absorbing polymer. DAB is produced when amino acids decompose, and because it exists in nature, we considered it to be a material that would reduce the environmental impact of
PAsp synthesis.

\section{EXPERIMENTAL}

\subsection{Materials}

L-aspartic acid was used as the raw material for PAsp and $85 \%$ phosphoric acid was employed as the acid catalyst. Mesitylene was used as the heat and dispersion medium, and N, N-dimethylformamide (DMF) was utilized as the solvent for converting PSI into cross-linked PSI.

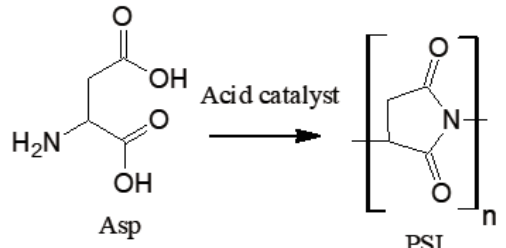

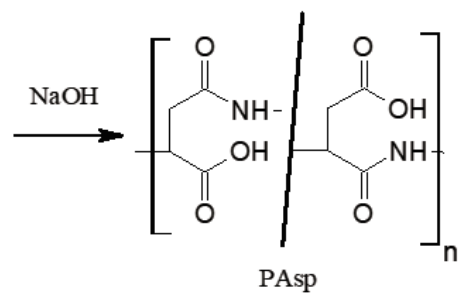

Fig. 1 Synthesis of PAsp.

\subsection{Synthesis of water-absorbing polymers}

PSI was synthesized by stirring and heating L-aspartic acid $(25 \mathrm{~g}), 85 \%$ phosphoric acid $(1.5 \mathrm{~mL})$, and mesitylene $(90 \mathrm{~mL})$ in a two-neck round-bottom flask [6]. Water was removed using a Dean-Stark trap while refluxing. After $4.5 \mathrm{~h}$, the solvent was removed, and the mixture was washed thrice with methanol $(100 \mathrm{~mL})$ and reverse osmosis (RO) water $(100 \mathrm{~mL})$. The product was dried in an oven at $100{ }^{\circ} \mathrm{C}$ to obtain crude PSI.

The crude PSI was added to DMF $(100 \mathrm{~mL})$ and stirred. After $4 \mathrm{~h}$, the solid was removed and the solution was placed in ethanol $(100 \mathrm{~mL})$ to precipitate the PSI. The solvent was removed and PSI was washed thrice with RO water $(100 \mathrm{~mL})$. The solid was dried in an oven at $100{ }^{\circ} \mathrm{C}$ 
to yield pure PSI.

The pure PSI $(5.0 \mathrm{~g})$ was added to DMF $(100 \mathrm{~mL})$ and stirred for $4 \mathrm{~h}$, after which a DAB solution (with varying $\mathrm{DAB} /$ polymer ratios) in DMF $(3.0 \mathrm{~mL})$ was gradually added. After $2 \mathrm{~min}$, the mixture was added to ethanol (200 $\mathrm{mL}$ ) to precipitate the cross-linked PSI. The solvent was removed, the precipitate was washed with ethanol $(200$ $\mathrm{mL}$ ), and was then dried in an oven at $100{ }^{\circ} \mathrm{C}$ to yield cross-linked PSI.

The cross-linked PSI was added to RO water $(100 \mathrm{~mL})$ and stirred. The $\mathrm{pH}$ of the solution was adjusted to 9-11 using $2 \mathrm{~mol} / \mathrm{L} \mathrm{NaOH}$. After $1 \mathrm{~h}$, the solvent was removed to yield cross-linked PAsp. The gel was washed with a large amount of RO water and dried in an oven at $60{ }^{\circ} \mathrm{C}$.

All the obtained synthesized products were characterized through Fourier transform infrared (FT-IR) spectroscopy ( $\mathrm{KBr}$ method).

\subsection{Measurement of water absorption}

Water absorption was measured with reference to JIS K 7223 [7]. Dried, cross-linked PAsp $(0.20 \mathrm{~g})$ was accurately weighed and placed in a nylon mesh bag (200 $\mathrm{mm} \times 100 \mathrm{~mm}$ ). The bottom $150 \mathrm{~mm}$ of the bag was immersed in RO water, and after $3 \mathrm{~h}$ and then $24 \mathrm{~h}$ the bag was drained for $10 \mathrm{~min}$ and the mass was measured.

The amount of water absorption was calculated using the following equation:

$$
W=\frac{b-c-a}{a}
$$

Where $W$ is the amount of water absorption ( $\mathrm{g} / \mathrm{g}), a$ is the mass of the sample (g), $b$ is the mass of the tea bag with the sample immersed for a specified time and then drained $(\mathrm{g})$, and $c$ is the average mass $(\mathrm{g})$ of the tea bag without the sample.

\subsection{Measurement of water absorption rate}

Water absorption rate measurements were performed with reference to JIS K 7224 [8]. Approximately $50 \mathrm{~mL}$ of RO water was placed in a $100-\mathrm{mL}$ beaker. A stirrer chip (central part $\varphi 8 \mathrm{~mm}$, both ends $\varphi 7 \mathrm{~mm}, 30 \mathrm{~mm}$ ) was placed in the beaker and stirred at $600 \mathrm{rpm}$. After confirming that the water formed a stable vortex, $2.0 \mathrm{~g}$ of the cross-linked PAsp was added. The time from when the cross-linked PAsp was added until the stirrer chip was covered with the test solution was measured, and from this the water absorption rate was calculated using the following equation:

$$
V=\frac{\frac{M}{a}}{t}
$$

Where $V$ is the water absorption rate $(\mathrm{g} / \mathrm{g} / \mathrm{s}), M$ is the mass $(\mathrm{g})$ of the test solution, $a$ is the mass $(\mathrm{g})$ of the sample, and $t$ is the time to the end point.

\subsection{Measurement of water retention}

The water retention test was conducted by placing the cross-linked PAsp and black clay into separate nylon mesh bags [9]. The lower $150 \mathrm{~mm}$ of each bag was immersed in RO water, and after $3 \mathrm{~h}$, the bag was drained for $10 \mathrm{~min}$. The polymer that had absorbed up to $4 \mathrm{~mL}$ of water was placed in a $15-\mathrm{mL}$ falcon tube and then weighed. It was stored at $20^{\circ} \mathrm{C}$ for 20 days, during which the mass was arbitrarily measured. The water content of each sample was calculated using the following equation:

$$
C=\frac{e-g-f}{e-g} \times 100
$$

Where $C$ is the water content (\%), $e$ is the mass (g) of the falcon tube containing the absorbed sample, $g$ is the mass $(\mathrm{g})$ of the falcon tube, and $f$ is the mass $(\mathrm{g})$ of the dried sample. The water retention was then determined based on the change in the water content with time.

2.6 Germination test of soil containing water-absorbing polymer

Black clay $(170 \mathrm{~g})$ and river sand $(320 \mathrm{~g})$ were separately mixed with cross-linked PAsp $(5.0 \mathrm{~g}$, $\mathrm{DAB} /$ polymer $\mathrm{mol} / \mathrm{mol}=20 \%$ ) and placed in disposable cups with a hole in the bottom [10].

Each sample was immersed in $\mathrm{RO}$ water $(200 \mathrm{~mL})$ for $30 \mathrm{~min}$ and then drained for $10 \mathrm{~min}$. Subsequently, the soil was flattened on a tray $(255 \mathrm{~mm} \times 175 \mathrm{~mm} \times 45$ $\mathrm{mm})$. Radish sprout was the plant used for the germination tests because it is a plant that grows only in water and can be harvested in about 10 days. Radish sprout seeds (100) were sown in each soil type. The germination rate of the radish sprouts was calculated daily for 10 days using the following equation:

$$
G=\frac{g}{S} \times 100
$$

Where $G$ is the germination rate (\%), $g$ is the number of germinated seeds and $s$ is the number of seeds sown. After the 10 days, the lengths of the radish sprout shafts were measured.

\section{RESULTS AND DISCUSSION}

3.1 Synthesis of water-absorbing polymer

The cross-linked PSIs with different DAB/polymer ratios were successfully synthesized in yields of $95 \%$ or more (Table I). The yield when the ratio was $15 \%$ actually exceeded $100 \%$. These high yields were likely because of the cross-linked structure introduced into the PSIs and the reduced solubility in the solvent. Kishimoto et al. [11] reported the insufficient removal of DMF during ethanol washing to be the reason behind the high yields.

When the cross-linked PSI was hydrolyzed with an alkali, the compounds with a $\mathrm{DAB} /$ polymer molar ratio of $5-15 \%$ were completely dissolved in the solution and the cross-linked PAsp could not be recovered. This can be ascribed to poor insolubilization resulting from the cross-linking and insufficient cross-linking between the molecules. This is supported by the yield of PAsp increasing as the ratio of $\mathrm{DAB} /$ polymer increases (Table I).

Each compound was analyzed by FT-IR. The spectra of PSI and the cross-linked PSIs (Fig. 2a) show a broad absorption at $3700-3200 \mathrm{~cm}^{-1}$, a smaller absorption at $3000 \mathrm{~cm}^{-1}$, and another band at $1700 \mathrm{~cm}^{-1}$, which respectively correspond to the $-\mathrm{CONH}$ stretching, $\mathrm{COOH}$ stretching, and $-\mathrm{C}=\mathrm{O}$ stretching vibrations of the five-membered imide ring [12]. PSI normally does not 
Table I Synthesis results of each compound

\begin{tabular}{ccc}
\hline Compounds & $\begin{array}{c}\text { DAB/Polymer } \\
(\mathrm{mol} / \mathrm{mol})\end{array}$ & $\begin{array}{c}\text { Yield } \\
(\%)\end{array}$ \\
\hline PSI & $0 \%$ & 48 \\
\cline { 1 - 1 } PAsp & $0 \%$ & 85 \\
& $5 \%$ & 95 \\
Cross-linked & $10 \%$ & 98 \\
PSI & $15 \%$ & 106 \\
& $20 \%$ & 99 \\
& $25 \%$ & 97 \\
Cross-linked & $30 \%$ & 97 \\
PAsp & $20 \%$ & 53 \\
& $25 \%$ & 74 \\
\hline
\end{tabular}

have $-\mathrm{COOH}$ and $-\mathrm{CONH}$ groups; however, Nakato et al. [13] reported that PSI obtained by thermal polymerization is derived from a ring-opened structure or a branched structure that contain a $-\mathrm{CONH}$ and $-\mathrm{COOH}$ group. Therefore, these absorptions are observed. In addition, the absorption at $3070 \mathrm{~cm}^{-1}$ present in the spectra of PSI with $\mathrm{DAB} /$ polymer ratio of $10-30 \%$ are considered to represent a - $\mathrm{CH} 2$-stretching vibration. The intensity of this band increases as the amount of the cross-linking agent increases, indicating that it is derived from DAB. This confirms that DAB was successfully introduced into the PSI molecule.

The FT-IR spectra for PAsp and cross-linked PAsp (Fig. 2b) show a broad absorption at $3600-3200 \mathrm{~cm}^{-1}$ and two absorptions at $1600 \mathrm{~cm}^{-1}$ and $1400 \mathrm{~cm}^{-1}$. These are attributed to - $\mathrm{CONH}$ stretching, - $\mathrm{CONH}$-bending angle, and -COO- asymmetric/symmetric stretching vibrations, respectively [12]. The two absorptions at $3000 \mathrm{~cm}^{-1}$ in the spectrum of PAsp with $25-30 \%$ of cross-linking agent were considered to represent the stretching vibrations of - $\mathrm{CH} 2$ - from DAB

\subsection{Measurement of water absorption}

Water absorption measurements of the cross-linked PAsp (Table II) confirmed that the amount of water absorbed by PAsp after $3 \mathrm{~h}$ and after $24 \mathrm{~h}$ increased as the cross-linking agent decreased. In general, the water absorption capacity of a water-absorbing polymer can be expressed by the following formula: [14]

$$
W=\frac{I+A}{C}
$$

Where $W$ is the water absorption capacity, $I$ is the ion osmotic pressure, $A$ is the affinity of between the polymer and water, and $C$ is the crosslink density. As can be seen from the equation, as $A$ increases $W$ also increases. A higher $A$ value indicates that the ionic functional groups in the polymer have high densities. PAsp is cross-linked by the formation of an amide bond between the carboxyl group of PAsp and the amino group of DAB. Specifically, as the cross-linking agent increases, the number of carboxyl groups decreases and the density of the ionic functional groups in the polymer decreases. Moreover, $W$

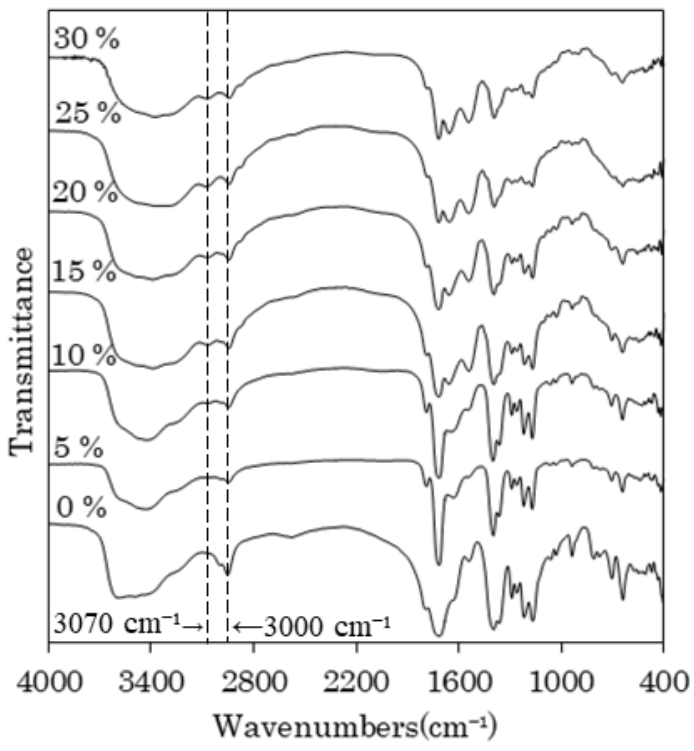

(a)

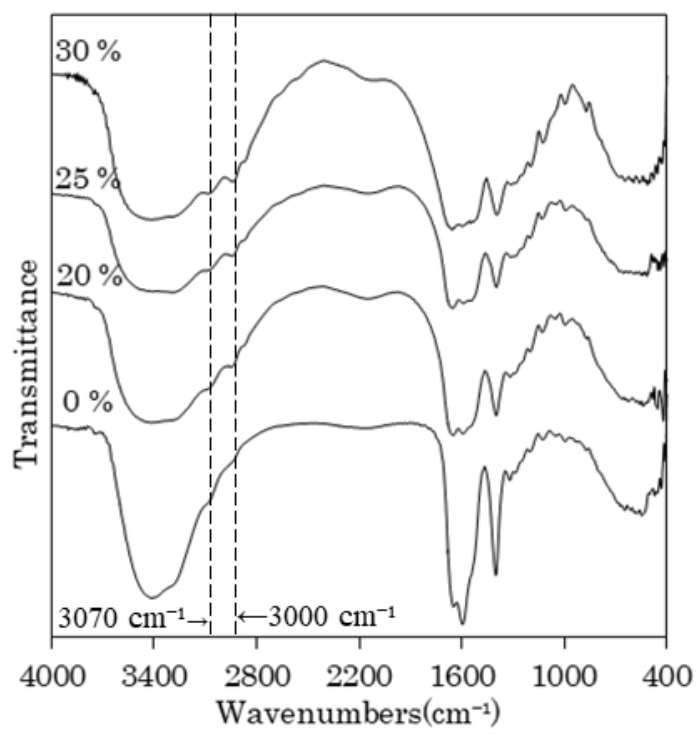

(b)

Fig. 2 Fourier transform infrared (FT-IR) spectra of (a) PSI and cross-linked PSIs and (b) PAsp and the crosslinked PAsps. The percentages listed in the figures represent the $\mathrm{DAB} /$ polymer ratios.

Table II. Water absorption of the cross-linked PAsps.

\begin{tabular}{ccc}
\hline \multirow{2}{*}{$\begin{array}{c}\text { DAB/Polymer } \\
(\mathrm{mol} / \mathrm{mol})\end{array}$} & \multicolumn{2}{c}{$\begin{array}{c}\text { Amount of water absorption } \\
(\mathrm{g} / \mathrm{g})\end{array}$} \\
\cline { 2 - 3 } & $3 \mathrm{~h}$ & $24 \mathrm{~h}$ \\
\hline $20 \%$ & 28.8 & 29.7 \\
$25 \%$ & 23.2 & 23.5 \\
$30 \%$ & 17.2 & 19.2 \\
\hline
\end{tabular}

is low when the $C$ is high. Therefore, the amount of water absorption would increase as the amount of the crosslinking agent decreases. 
3.3 Measurement of water absorption rate

As described in section 3.2, greater amounts of crosslinking agents reduce the number of carboxyl groups, which are hydrophilic. Therefore, both $A$ and the water absorption rate would decrease as more cross-linking agent was added. This was confirmed upon determining the water absorption rates for the cross-linked PAsps with $\mathrm{DAB} /$ polymer ratios of $20-30 \%$ (Table III).

Table III Water absorption rates of the cross-linked PAsps.

\begin{tabular}{cc}
\hline $\begin{array}{c}\mathrm{DAB} / \text { Polymer } \\
(\mathrm{mol} / \mathrm{mol})\end{array}$ & $\begin{array}{c}\text { Water absorption rate } \\
(\mathrm{g} / \mathrm{g} / \mathrm{s})\end{array}$ \\
\hline $20 \%$ & 11.0 \\
$25 \%$ & 4.8 \\
$30 \%$ & 1.7 \\
\hline
\end{tabular}

\subsection{Measurement of water retention}

Changes in the water content of black soil with and without the cross-linked PAsps were observed over 20 days (Fig. 3). The black soil without the polymer showed a decrease in water content of $4.5 \%$. However, the soil with the cross-linked PAsps (all DAB/polymer ratios) presented a water content reduction of $1 \%$ or less. This suggests that the cross-linked PAsps can ensure water retention in soil because of their high water retention capability.

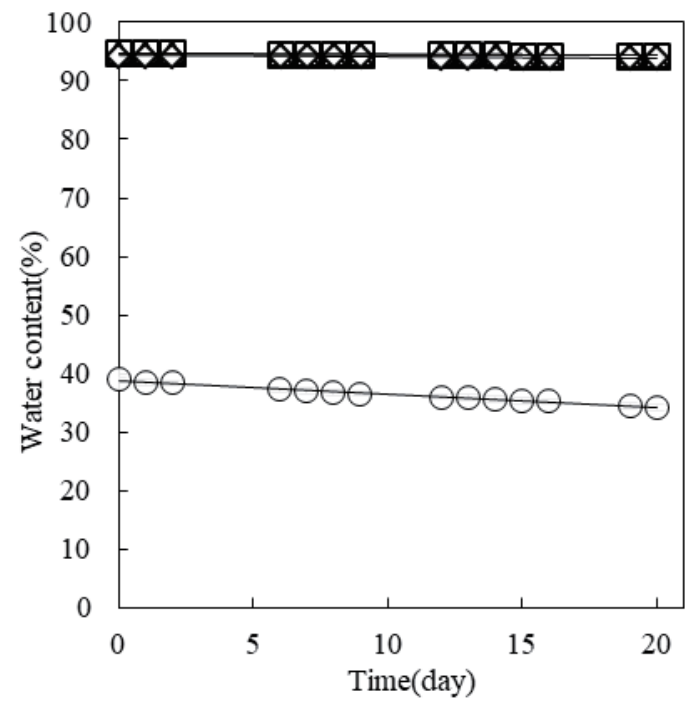

Fig. 3 Change in water content of black soil over time with $20 \%$ cross-linked PAsp ( $\square$ ), $25 \%$ cross-linked PAsp $(\triangle), 30 \%$ cross-linked PAsp $(\diamond)$ and without crosslinked PAsp $(\bigcirc)$.

3.5 Germination test of soil with water-absorbing polymer

Using a super absorbent polymer as a greening material has reportedly promoted plant germination but has also caused germination damage under various conditions, such as polymer composition [3]. Therefore, changes in the germination rate of the radish sprouts were observed over time (Fig. 4). The germination rate of the soil with the cross-linked PAsp was equal to or higher than that of the soil without PAsp. This confirms that cross-linked PAsp can be used in soil without disrupting the germination of radish sprouts.

The length of the radish sprout leaves were measured after 10 days of germination in each soil (Fig. 5). When grown in the black soil without the polymer the longest shaft was measured to be $6-12 \mathrm{~mm}$, which increased to $12-18 \mathrm{~mm}$ when the polymer was present. When grown in river sand the addition of the cross-linked PAsp did not affect the shaft length, the longest of which was measured to be $6-12 \mathrm{~mm}$. Furthermore, the axial length distribution varied more when the cross-linked PAsp was not added than when it was added. These results show that crosslinked PAsp in black soil increases the shaft length of radish sprouts but only stabilizes shaft length when added to river sand.

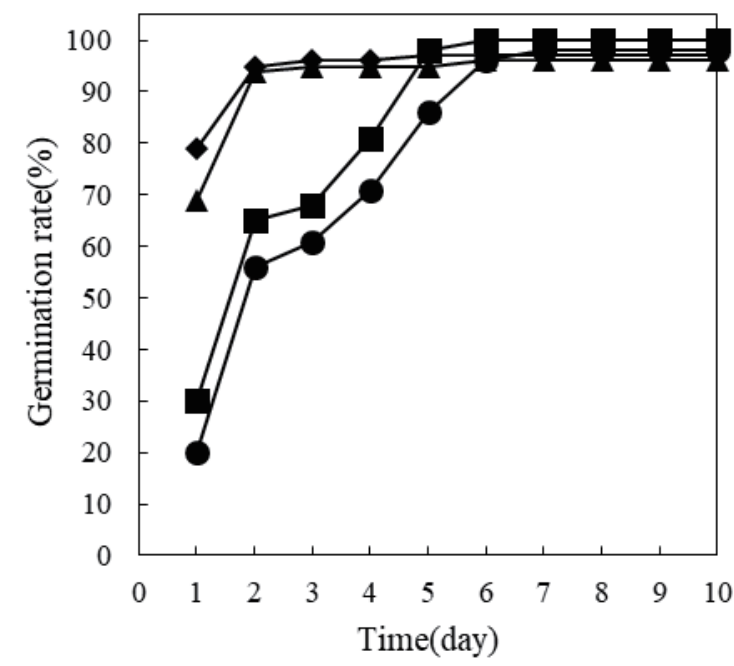

Fig. 4 Germination rates of white radish sprouts in black soil (๑), in black soil with cross-linked PAsp ( $\square)$,in river sand $(\mathbf{\Lambda})$, and in river sand with cross-linked PAsp ( $)$.

\section{CONCLUSION}

In this study, PAsp was cross-linked with $\mathrm{DAB}$ to obtain a superabsorbent polymer. The best water absorption properties were observed when the DAB/polymer $(\mathrm{mol} / \mathrm{mol})$ ratio was $20 \%$. Black soil showed increased water retention when the cross-linked PAsp was added, and germination tests of radish sprouts in black soil and river sand demonstrated that the polymer promoted elongation growth of the plant in black soil and suppressed axial length dispersion in the river sand.

These results show that cross-linked PAsps can be successfully employed as a greening material and are made from materials with low environmental impact. 

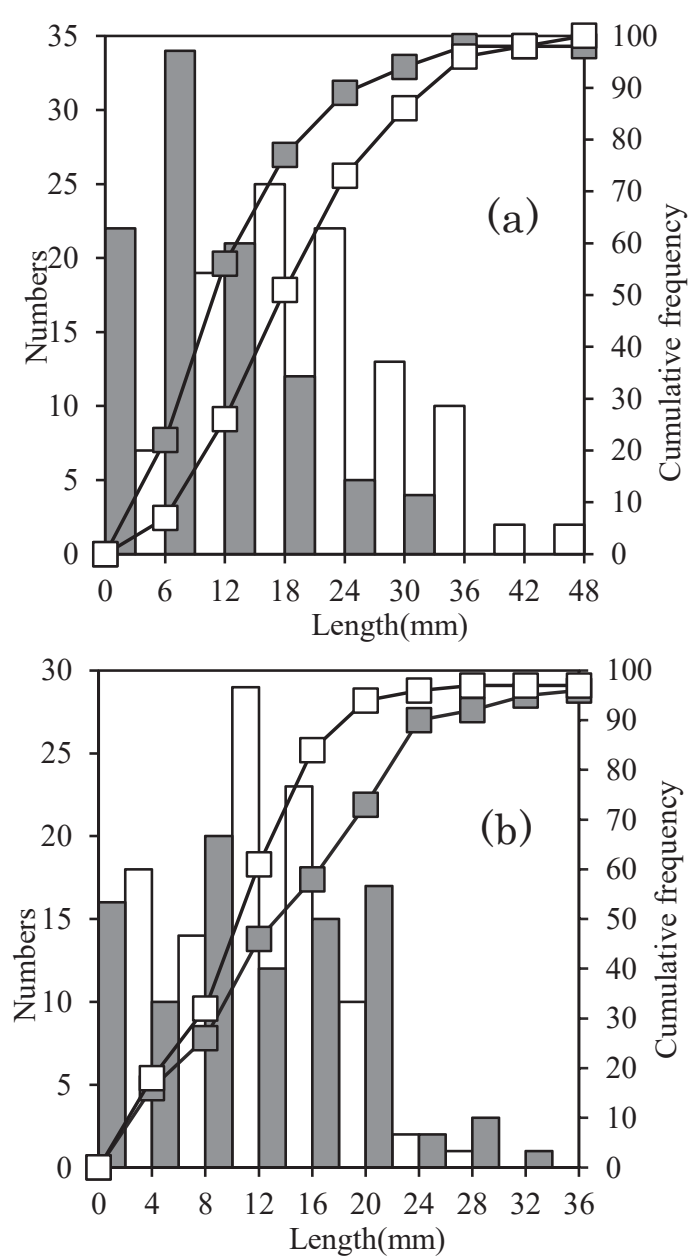

Fig. 5 Lengths of radish sprouts germinated in (a) black soil ( : 98) and black soil with cross-linked PAsp ( $\square: 100)$ and (b) in river sand ( $\square: 96)$ and river sand with cross-linked PAsp ( $\square:$ 97).

\section{REFERENCES}

[1] R. Yoshida, "koubunshi Gel", Kyoritu Shuppan Co. Press, Japan, (2004)pp.5-9,76-78.

[2] S. Matsumura, SEN'I GAKKAISHI, 52, 23-27(1996).

[3] Y. Sugimura and Y. Yamadera, Ryokkakougijutsu, 9, 11-12(1983).

[4] M. Tomida andT. Nakatou, Koubunsi, 50, 393 (2001).

[5] M. Enna, Kagaku to Kyouiku, 53, 22 (2005).

[6] M. Tomida, T. Nakato, S. Matsunami, and T. Kakuchi, Polymer, 38, 4734-4736 (1997).

[7] JIS K 7223 (1996).

[8] JIS K 7224 (1996)

[9] A. Ninhei, E. Ota, A. Kobayashi, T, Yuzawa. and M. Yuzawa, Tochigi Sanngyou Gijyutsu Center Kenkyuhoukoku, 13, 22 (2016).

[10] T. Kimura, A. Misawa, T. Ochiai, and S. Ito, Shibakusa Kenkyu, 16, 5-6 (1987)

[11] Y. Kishimoto, K. Tsuda, M. Miyjima and S. Umeda, Journal of the National institute of Technology, Asahikawa College, 41, 75 (2004).

[12] M. Hesse, H. Meier, and B, Zeeh, "Spectroscope Methods in Organic Chemistry", Tokyo Kagaku Doujin Co. Press, Japan, (2010) pp. 41-53.

[13] T. Nakato, M. Yoshitake, K. Matsubara, and M.
Tomida, Macromolecules, 31, 2109-2110 (1998).

[14] F. Masuda and K. Tanaka, Nihon Kasei Gakkai Shi, 40, 721 (1989).

(Received March 27, 2020; Accepted August 24, 2020; Published Online October 1, 2020) 